\title{
Culture écologique : étude exploratoire de la participation de médias québécois à la construction de représentations sociales de problèmes écologiques \\ Participation of Quebec Media in the Construction of the Social Repr sentations of Ecological Problems: An Exploratory Study
}

\section{Carmen GENDRON et Brigitte DUMAS}

Volume 23, numéro 1, printemps 1991

Savoirs institués, savoirs informels

URI : https://id.erudit.org/iderudit/001292ar

DOI : https://doi.org/10.7202/001292ar

Aller au sommaire du numéro

Éditeur(s)

Les Presses de l'Université de Montréal

ISSN

0038-030X (imprimé)

1492-1375 (numérique)

Découvrir la revue

Citer cet article

GENDRON, C. \& DUMAS, B. (1991). Culture écologique : étude exploratoire de la participation de médias québécois à la construction de représentations sociales de problèmes écologiques. Sociologie et sociétés, 23(1), 163-180.

https://doi.org/10.7202/001292ar

\section{Résumé de l'article}

Cet article questionne la participation des médias à la construction des représentations sociales de l'écologie dans la société québécoise. Le contenu des textes de trois revues québécoises et d'une émission radiophonique y est analysé à travers une problématique des représentations sociales, laquelle fait une large place aux concepts d'objec-tivation et d'ancrage. Par l'objectivation, les informations sont sélectionnées, triées et organisées en vue de présenter une vision du monde. Les mécanismes d'ancrage scrutent les objectifs, l'intention et la perspective qui rendent les éléments compréhensibles les uns par rapport aux autres. L'article identifie ainsi les problèmes et solutions proposées par les médias et lie leurs choix aux perspectives qui fondent le discours pour dégager le rôle de producteurs de sens et de savoir qu'assument les médias en matière d'écologie.
Tous droits réservés ㄷ Les Presses de l'Université de Montréal, 1991
Ce document est protégé par la loi sur le droit d'auteur. L'utilisation des services d’Érudit (y compris la reproduction) est assujettie à sa politique d'utilisation que vous pouvez consulter en ligne.

https://apropos.erudit.org/fr/usagers/politique-dutilisation/ 


\title{
Culture écologique: étude exploratoire de la participation de médias québécois à la construction de représentations sociales de problèmes écologiques
}

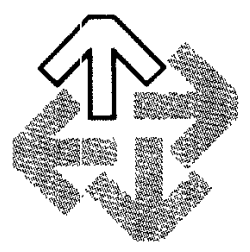

\author{
BRIGITTE DUMAS \\ et CARMEN GENDRON
}

Dans la société québécoise, l'enseignement de l'écologie à l'intérieur de programmes scolaires est une pratique récente à laquelle seuls les moins de vingt ans ont pu goûter. Pourtant, dans l'ensemble du public, la connaissance des questions écologiques apparaît assez importante pour quiconque écoute les lignes ouvertes à la radio ou s'intéresse au courrier des lecteurs. Par quelles voies ces connaissances circulent-elles? Comment une opinion publique se forme-t-elle ainsi au fil des années? Les acteurs utilisent-ils les connaissances instituées ou se forment-ils simplement une opinion superficielle sur l'écologie? Telles sont les questions que nous avons dégagées lors de la définition de notre projet d'étude sur les représentations sociales des problèmes écologiques.

Puisque les savoirs écologiques du public ne passent pas par les milieux d'enseignement institutionnalisés, nous avons orienté notre recherche vers les médias. Les médias, en raison de leur rôle de communication, contribueraient à créer une culture écologique ou un «esprit de l'écologisme» au Québec. Notre hypothèse veut que les mass media procèdent à un bricolage original qui retient des éléments du discours scientifique tout en fonctionnant selon une logique pragmatique de la pertinence: il y aurait donc processus d'appropriation de la logique scientifique suivant une logigue qui serait celle des représentations sociales.

Nous avons retenu pour les fins de notre analyse des médias écrits qui se donnent d'emblée une vocation de vulgarisation scientifique: il s'agit des revues Québec Science. Franc Nord et Écologie, auxquels s'ajoute l'émission radiophonique Aujourd' hui la science.

Afin d'analyser cet «esprit de l'écologisme», nous avons choisi d'appliquer à ces discours une problématique des représentations sociales, car elle nous permet de reconstituer la spécificité des systèmes cognitifs intimement liés à l'expérience.

\section{LES REPRÉSENTATIONS SOCIALES}

Les représentations sociales ont fait l'objet de nombreuses études en sociologie et en psychologie. Différentes approches liées aux champs de pratique de ces disciplines ont émergé et donnent des interprétations souvent divergentes de ce qu'est une représentation sociale. Il revient à la psychologie sociale d'avoir la première cherché à définir la relation entre un sujet qui s'approprie le social et une société, considérée comme un déjà-là, qui 
interfère dans le libre arbitre dont jouirait le sujet. Ces approches ont donné lieu à des analyses telles que les effets des représentations sur le comportement, les schèmes organisateurs des représentations de groupe, la structure des représentations (Abric, 1984; Codol, 1970; Abric et Vacherot, 1975; Flament, 1981; Abric et Mardella, 1974), etc. Elles ont aussi suscité des études sur la relation entre représentations et assimilation de connaissances (Ackerman, Zigouris, 1973; Albertini, 1984; Grize et al., 1987), etc. Ces dernières approches ont particulièrement contribué à la recherche d'une synthèse entre l'approche sociologique et l'approche cognitive dans l'étude des représentations sociales. Elles restent pourtant centrées sur l'aspect individuel de la représentation sociale: «Une représentation est l'expression de la manière dont le monde, l'environnement, le signal est reconstruit par le système cognitif d'un individu. C'est l'expression de la réinterprétation de ce qui est perçu comme réalité extérieure ou intérieure.» (Albertini, 1984, p. 310.) L'influence de l'approche cognitiviste y est manifeste. Les cognitivistes semblent s'entendre sur l'existence de stocks minimaux relativement stables d'informations mémorisées (mémoire générale permanente). Ces stocks sont décrits tantôt sous forme d'images mentales ou verbales, tantôt sous forme de schèmes qui seraient la «structure du contenu d'un domaine particulier» (La Ménardière, 1985, p. 243). Le schème est «un ensemble structuré et modifiable d'informations représentant des concepts génériques conservés dans la mémoire. Les schèmes représentent le contenu de notre expérience: relations entre objets, situations, événements, suites habituelles d'événements» (Glaser, 1988, p. 27).

Si de telles analyses restent fondamentales pour notre compréhension du fonctionnement du système cognitif individuel, elles demeurent toutefois ambiguës et incomplètes lorsqu'elles prétendent rendre compte du caractère social des représentations. En fait, dans ces approches, le caractère social réside essentiellement dans l'intégration de catégories socialement organisées. La limitation inhérente à l'approche cognitiviste des représentations sociales tient au fait qu'elle ne considère les représentations qu'en ce qu'elles sont déjà constituées en produits sous forme de déterminants sociolinguistiques. Cette limitation est mise en évidence par J.-P. Di Giacomo lorsqu'il critique les psychologues sociaux, qui ont ramené la représentation sociale au niveau d'un contenu mental individuel suscité par la perception d'un objet: «À des degrés divers, une telle réduction est perceptible chez Herzlich (1972), Doise (1976) et Le Bouedec (1979) tout comme dans la plupart des recherches expérimentales déjà citées. Ces auteurs parlent indifféremment d'images, de stéréotypes ou de représentations et confondent les représentations sociales avec les processus représentationnels des individus. » (Di Giacomo, 1981; p. 399.)

C'est en fait à Moscovici que l'on doit une approche des représentations sociales qui définit l'interdépendance et l'interfécondation entre individu et société; il a mis en évidence le processus par lequel la construction de représentations sociales équivaut à la construction d'une pensée sociale. Dans cette approche, la communication devient le fondement d'une identité sociale, le fondement d'une culture commune. «The reason representations are social is not only that they have a common object or that they are shared. It is also that in our society they have a certain autonomy and are the outgrowth of a codified know-how that enjoys an undeniable authority. (Moscovici, 1988, p. 225.)

Jodelet (1989) a distingué avec justesse trois niveaux différents dans la conception du processus de communication chez Moscovici. D'abord, à un premier niveau, on assiste à l'émergence des représentations puis, à un second niveau, à la formation des représentations, enfin, à un troisième niveau, s'établit un rapport entre représentation et conduite sociale. Comme le dit Jodelet, la communication chez Moscovici revêt ainsi un double statut: elle rend possible un agir et détermine la représentation.

Depuis son étude de 1961 sur les représentations sociales de la psychanalyse, Moscovici n'a cessé d'insister sur la nécessité de traiter les représentations sociales comme des processus cognitifs et symboliques sui generis, c'est-à-dire ayant une autonomie et une efficace propres dans la construction de la réalité sociale. Il considère que la formation des représentations est tributaire de deux processus, l'objectivation et l'ancrage, qui lient l'acti- 
vité cognitive du sujet aux conditions sociales. Ces processus ne manquent pas d'être relativement complexes. On en présentera ici les caractéristiques les plus essentielles. Le mécanisme d'objectivation peut rendre compte du processus par lequel les informations et les connaissances disponibles sont sélectionnées, triées et organisées pour présenter une vision du monde: le monde comme objet. Les mécanismes d'ancrage, pour leur part, réfèrent aux objectifs d'intervention, à l'intention, à la perspective qui rendent les divers éléments compréhensibles les uns par rapport aux autres, et efficaces par rapport à un champ de pratique sociale. L'objectivation et l'ancrage sont solidaires dans le processus d'élaboration des représentations sociales car, comme Jodelet (1989) l'a si justement noté: «En amont, l'ancrage enracine la représentation et son objet dans un réseau de significations qui permet de les situer en regard des valeurs sociales et de leur donner cohérence. En aval de la formation représentative, l'ancrage sert à l'instrumentalisation du savoir en lui conférant une valeur fonctionnelle pour l'interprétation et la gestion de l'environnement. Il se situe alors en continuité avec l'objectivation.» (Jodelet, 1989, p. 56.)

C'est à partir de ces deux mécanismes que nous analysons le travail qu'opèrent les médias dans la culture. Les médias procèdent à une objectivation du monde en utilisant les connaissances disponibles dans la société.

\section{MÉTHODES D'INVESTIGATION DES REPRÉSENTATIONS SOCIALES DES PROBLÈMES ÉCOLOGIQUES}

Notre recherche visait à mettre en évidence les connaissances produites par différents médias en ce qui a trait à l'écologie. Nous avons choisi d'analyser deux médias qui se donnent d'emblée un mandat écologiste (Franc Nord et Écologie) et deux médias qui se définissent d'abord par leur fonction de vulgarisation scientifique (Québec Science et magazine radiophonique Aujourd'hui la science). Notre objectif était de mettre à jour des connaissances observables au moyen de l'objectivation et de définir l'intention qui articule les connaissances entre elles au moyen de l'ancrage. Il ne s'agissait pas pour nous d'établir la démarcation entre science et non-science mais de décrire des styles médiatiques qui pourraient renvoyer à un «esprit de l'écologisme». À ce titre, notre méthode est essentiellement descriptive et inductive. Ce travail nous a permis, dans un premier temps, de repérer des traits distinctifs du discours médiatique pour ensuite construire des catégories descriptives par agrégation d'unités de sens.

Au chapitre de l'objectivation, nous avons procédé par une analyse de contenu d'articles de revues et d'émissions de radio. Nous avons retenu tous les textes qui problématisaient des questions environnementales. Dans un premier temps, nous avons cherché à définir quel était le problème principal dont traitait l'article. L'analyse des mécanismes d'ancrage a été réalisée à partir de questions sur les enjeux identifiés par les revues, d'une part, et sur les objectifs et style du discours, d'autre part. Il ne s'agissait plus ici de mettre en évidence des contenus de discours, mais plutôt leur modalité d'organisation, de façon à faire émerger l'intention qui les fonde.

\section{REPRÉSENTATIONS DES PROBLÈMES ÉCOLOGIQUES DANS LES MÉDIAS QUÉBÉCOIS}

\section{PROBLÈMES ET SOLUTIONS}

Au total, 19 articles de revues ${ }^{1}$ et 9 émissions radiophoniques ont été analysés ${ }^{2}$. La

1. Les articles analysés couvrent pour Écologie le vol. 1, n"2 de décembre 1989, pour Québec Science le vol. 25. $n^{\circ} 4$ de janvier 1990 et, pour Franc Nord le numéro hors série 4, janvier 1990. Les émissions radiophoniques d'Aujourd' hui la science ont été diffusées entre le 28 janvier ct le 25 mars.

2. Certains textes des revues ont été éliminés, ne correspondant pas à la définition de l'écologie que nous avons proposée ou ne pouvant être décrits comme des articles. Certains ne constituaient que des entrefilets 
compilation des résultats a montré que le traitement des problèmes s'articule autour de quatre thèmes: le premier, très général, traite du rapport de l'homme à la nature sous la thématique de la planète en danger. Les trois autres s'intéressent à des aspects spécifiques, soit : a) pollution et déchets; b) gaspillage des ressources; c) développement durable. Ces thèmes constituent le noyau central des problèmes environnementaux, et l'analyse de leurs composantes nous donne accès au processus d'objectivation propre aux représentations sociales que construisent les médias. De même, les solutions proposées sont tantôt institutionnelles en ce qu'elles renvoient au «système», tantôt individuelles en ce qu'elles renvoient au «mode de vie». L'examen des solutions proposées et des problèmes identifiés par les médias permet d'appréhender les modalités par lesquelles ceux-ci s'approprient la problématique environnementale dans son ensemble, le processus par lequel ils en font une réalité objective.

Nous avons précédemment identifié quatre thèmes recouvrant l'ensemble de la problématique générale de l'écologie dans les médias. Si les articles définissent un problème principal, ils n'en traitent pas moins d'un ensemble de sous-problèmes qui ont pour rôle d'illustrer, de rendre plus concret le problème principal. Ainsi, il ne faut pas s'étonner de rencontrer dans l'identification d'un problème comme la planète en danger l'utilisation de problématiques liées à la pollution ou au gaspillage... En fait, ces quatre thèmes s'interpellent les uns les autres. La classification permet, d'une part, d'identifier les priorités que se donnent les revues et, d'autre part, de mettre en évidence l'articulation de la pensée écologique selon les thèmes proposés.

Les problèmes de pollution reçoivent le plus d'attention ( 22 documents), suivis du gaspillage des ressources (12), du thème de la planète en danger (3) et du développement durable (2). Les différents médias accordent une attention différente à chacun des thèmes. Ainsi, Franc Nord n'aborde que les questions de gaspillage des ressources (4/4) et Aujourd' hui la science s'intéresse quasi exclusivement à la pollution (8/9). Pour Écologie, c'est aussi la pollution qui occupe le plus d'espace (6/12), mais la revue traite aussi des risques pour la planète $(3 / 12)$, du gaspillage des ressources $(2 / 12)$ et du développement durable (1/12). Trois textes seulement proviennent de Québec Science. Ils traitent de la pollution (2) et du gaspillage (1).

La thématique de la planète en danger aborde de façon large et généreuse les questions écologiques. On s'interroge sur l'origine des problèmes qui menacent la planète dans son ensemble, sur les grandes orientations prises ou à prendre afin de faire face à ces problèmes. Trois articles, tous de la revue Écologie, en traitent. Le ton est alarmiste. On invoque la nécessité de comprendre et de s'impliquer face aux risques d'irréversibilité. La problématique Nord-Sud, au cour du débat, interpelle le gaspillage des ressources énergétiques et la pollution qu'il entraîne. On fait appel à la science, dont «les signaux restent contradictoires» (Écologie). On pointe du doigt les gouvernements et les industries qui ne bougent pas, ou qui ne bougent qu'une fois la population mobilisée. La revue insiste sur la faiblesse des moyens à la disposition des groupes écologistes et leur marginalisation dans la société. Sous cet angle, les problèmes sont vus de façon alarmiste, suivant une vision où s'opposent le Nord et le Sud, les gouvernements et les citoyens, les écologistes et les non-écologistes, les scientifiques entre eux... Les solutions sont aussi vastes et généreuses que les problèmes eux-mêmes. Il importe que de grands principes guident les décisions, qu'elles soient a) d'ordre administratif; le rôle et la place de chacun; b) d'ordre politique: des gouvernements qui veulent agir, une population mobilisée, des groupes écologistes efficaces; c) d'ordre économique : des budgets et des choix de production: d) d'ordre scientifique: de nouvelles sciences et l'interdisciplinarité.

La pollution et les déchets constituent des sous-produits de l'industrialisation dont l'origine est la sur-consommation. Voilà une idée universelle. Conséquemment, tous les domaines de la vie sont touchés, allant de la production d'énergie à la production de biens

non signés et d'autres ne présentaient que des exposés théoriques sur certaines espèces animales, végétales, ou encore sur l'art de cultiver son potager. 
et services en passant par la consommation domestique. Nous sommes tous responsables, diront les revues québécoises. Qu'on utilise l'électricité ou le pétrole, on met en danger l'environnement: monoxyde de carbone, hydrocarbures, ozone affectent la santé des végétaux, des animaux et des humains. Les pluies acides et l'effet de serre en sont les conséquences les plus néfastes. Pour faire face à ces problèmes, il importe, diront Écologie et Québec Science, de comprendre et de définir les tâches et les responsabilités des institutions et des groupes, notamment les institutions politiques, scientifiques et économiques. En matière de pollution, on tend à attendre beaucoup de la science, mais aussi et surtout à critiquer sa propension à imposer ses conclusions comme seules valables. La science est considérée comme n'étant pas neutre, elle véhiculerait des intérêts particuliers et ne doit par conséquent jouer qu'un rôle de conseiller.

En ce qui a trait aux questions de pollution, les propos de l'émission radiophonique Aujourd'hui la science sont essentiellement axés sur les activités des scientifiques. Les recherches sur la couche d'ozone, les moyens techniques d'assurer la dépollution de l'eau, les recherches concernant les plastiques biodégradables sont les thématiques développées. L'émission se veut critique. Elle signale les controverses. Elle remet fréquemment en cause les présumées relations de cause à effet qui lient des conséquences précises à un problème spécifique. Elle met en évidence les nouvelles technologies, leur spécificité et leur performance par rapport aux méthodes antérieures. Ici, les grands principes qui doivent guider

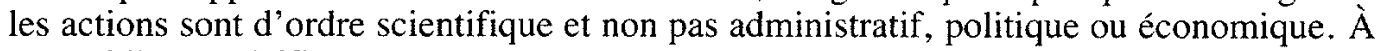
un problème spécifique, on propose une analyse spécifique: identifier le problème, établir les mesures, exécuter les recherches, développer les techniques appropriées, telles sont les questions à résoudre. On n'aborde pas les questions sociales, sauf si elles sont essentielles pour identifier la nature du problème.

La thématique du gaspillage des ressources aborde elle aussi les problèmes d'industrialisation et de surconsommation, non pas à travers la production de sous-produits, comme le fait l'approche pollution et déchets, mais plutôt à travers la protection des ressources existantes, leur remplacement et leur mise en valeur. Québec-Science, Écologie et Franc Nord y font tous référence. Dans les pages de Québec Science, nous explorons surtout le problème du gaspillage de l'énergie. Quant à Franc Nord, elle se préoccupe d'abord des ressources naturelles que compromettent l'agriculture, l'urbanisation et le développement industriel. Au chapitre des solutions au problème du gaspillage, les revues insistent particulièrement sur le développement de concepts plus appropriés pour définir les actions: l'efficacité énergétique est de cet ordre, le développement de projets visant à protéger sites, terrains et forêts également. L'action des groupes écologiques apparait déterminante pour résoudre les problèmes de gaspillage.

Le concept de développement durable, pour sa part, est au cœur d'un nouveau discours écologique qui tente de rallier des ennemis traditionnels : l'écologie et l'économie. Il est un discours écologique sur l'économie et un discours économique sur l'écologie dont l'objectif est d'assurer la croissance économique tout en préservant la planète. Ce concept dû à la Commission mondiale sur l'environnement (ONU, 1983) est actuellement repris au Québec et il a été l'enjeu d'un forum qui s'est tenu à l'automne 1990. Écologie considère que l'approche québécoise est trop axée sur l'éducation et néglige l'économie, que seuls des projets sans importance sont envisagés et que le tout souffre de nombreux délais.

Aujourd'hui la science aborde la même thématique dans le cadre du compte rendu d'un symposium tenu à Toronto, sur le thème, Le Nord et le Sud, un seul environnement. L'exposé insiste sur le changement d'orientation des programmes d'aide, lesquels seraient davartage orientés vers l'environnement. On y distingue la question environnementale du Nord (un problème de riches) de celle du Sud (un problème de pauvres). Dans ce contexte, l'émission insiste sur les solutions économiques, éducatives et technologiques.

Cet exposé des diverses conceptions avait pour but de saisir les façons dont les médias rendent objectives les questions environnementales. Nous avons noté l'existence de quatre thématiques principales, dont l'une plus générale, le danger pour la planète, occupe un espace 
relativement restreint dans le discours des médias. Les revues ont plutôt tendance à s'approprier les problématiques spécifiques, pollution ou gaspillage et de là, à généraliser les conséquences à l'ensemble de la planète.

Ainsi, lorsqu'une revue insiste sur la problématique du danger pour la planète, elle aborde les thèmes plus spécifiques de la pollution et du gaspillage, mais dans un objectif de généralisation. Elle cherche principalement à établir les liens entre les événements, entre les intervenants. La conséquence en est souvent un enfermement de la vie sociale, économique et politique dans une «marmite environnementale». À l'inverse, lorsqu'une émission oriente tous ses propos sur des questions spécifiques, telle que réduire la pollution de l'eau, elle dissèque la réalité elle-même en problèmes et en solutions spécifiques. Dès lors, tout semble pouvoir être résolu en appliquant la bonne méthode au bon problème. Cette approche correspondrait à ce que P. Dansereau appelle l'écologie-science, alors que la vision précédente peut davantage être rapprochée de l'éco-politique. Entre ces deux tendances, il existe une approche qui tente d'échapper à la généralisation à outrance tout comme à la tendance à démultiplier les problèmes. Cette approche serait davantage liée à l'écodéveloppement. La thématique du développement durable y serait associée. De façon évidente, l'émission Aujourd' hui la science s'inscrit dans l'approche écologie-science. Québec Science y souscrit à l'occasion mais se rapproche davantage de l'éco-développement. Franc Nord et Ecologie oscillent entre éco-développement et éco-politique.

Les représentations sociales de l'écologie apparaissent ainsi sous diverses formes et rendent compte d'un consensus à construire. Il est d'ailleurs difficile d'établir des dominantes puisque les thématiques s'interpellent, que les problèmes et solutions sont souvent similaires dans des approches distinctes. Le niveau d'imprécision sur la nature du risque est d'ailleurs exemplaire de cette absence de consensus. Que dix ans, diront certains articles. Les solutions émergent déjà, diront d'autres. Les médias ne sont pas en mesure de trancher et cette relative ignorance donne à leurs propos une image éclatée.

Devant un tel état de fait, il y a lieu de s'interroger sur l'utilisation des connaissances scientifiques par les médias. Deux explications sont possibles: ou bien les connaissances scientifiques actuelles sont incapables de rendre compte de la problématique environnementale, ou bien les journalistes sont coupés de ces connaissances. Il reste, cependant, que les connaissances d'ordre scientifique abondent dans les textes et qu'elles sont articulées aux connaissances de sens commun selon un style propre à chaque média. Ces connaissances scientifiques sont le plus souvent tirées des sciences naturelles. L'apport des sciences humaines telles que les analyses de comportements, sondages, études d'impact, analyses politiques, etc., y est quasi absent, ou à l'occasion dans le langage de quelque expert invité. C'est le média lui-même qui semble jouer le rôle d'analyste social.

Cette diversité des styles dans la production de l'information nous permet de repérer et de définir l'intention, intention que l'on peut lire dans les enjeux d'une part et dans les objectifs d'information, de sensibilisation et de légitimation donnés au discours. Nous sommes alors confrontés aux mécanismes d'ancrage des représentations.

\section{LES ENJEUX}

Généralement, les enjeux sont clairement identifiés par les auteurs, soit en introduction, soit en conclusion. L'identification et la classification des enjeux montrent que les orientations sont tantôt locales (société de conservation), tantôt globales (holisme environnemental). De plus, on y distingue une dichotomie entre la sphère matérielle (économique) et la sphère idéelle (politique et culturelle) de la société.

C'est de toute évidence la thématique de la société de conservation qui est au cœur du débat écologique au Québec. Toutes les revues et émissions de radio en traitent. Cette thématique regroupe deux catégories d'attentes. Les unes, orientées vers la sphère matérielle, occupent la plus grande partie du débat. Il s'agit donc de: 
— réconcilier progrès et protection de l'écosystème (Québec Science, Écologie);

- réduire la pollution et augmenter les profits (Québec Science);

- réduire la pollution par l'économie d'énergie (Québec Science);

- protéger les forêts contre les pressions du marché (Écologie);

- protéger les milieux naturels contre le développement économique (Franc Nord);

- réconcilier développement et écologie dans l'aide aux pays du Sud (Aujourd'hui la science).

L'autre catégorie de la thématique société de conservation est orientée vers la sphère idéelle. C'est ainsi qu'on insiste sur la nécessité de changer ou promouvoir des idées, de développer et promouvoir des organisations de défense. Il faut donc:

- irnpliquer tous les groupes sociaux (Écologie);

- discuter plus à fond du concept de développement durable (Écologie);

- inciter la population à comprendre et à coopérer (Franc Nord);

- développer l'intervention des écologistes dans la protection de la nature (Franc Nord).

Par ailleurs, 8 des 9 émissions d'Aujourd' hui la science se situent dans cette catégorie en plaçant les enjeux dans l'axe de l'évolution de la pensée scientifique. Deux ordres de préoccupations marquent l'émission:

1. La science à «la recherche du vrai» est l'une d'elles et s'exprime par des interrogations:

- sur la multiplicité des avenues, les difficultés de faire des choix en matière d'environnement et l'existence de conceptions fausses qui empêchent de poser correctement le problème;

- sur la démarche scientifique elle-même pour laquelle les relations causales ne peuvent êrre établies que si l'on a éliminé l'effet du hasard et envisagé toutes les explications;

- sur la constatation que la vérité ne peut advenir qu'en combattant les idées erronées, d'où la nécessité de la controverse.

2. La science «au service de...» est l'autre aspect qui débouche sur un désir de rendre la science applicable et utile à la résolution des problèmes. Il convient donc de :

- développer des recherches scientifiques pour résoudre un problème d'ordre politique;

- rendre la recherche fondamentale applicable en matière de dépollution;

- développer des sciences plus aptes à solutionner les problèmes liés à la pollution.

Seule la revue Écologie fait référence au holisme environnemental. On y fait état du défi de la survie planétaire devant la possible irréversibilité de la pollution mondiale. La sphère matérielle occupe une place dans ce débat et on souligne l'importance: 1) de se protéger contre le nucléaire qui menace la survie de la planète; 2) d'assurer une gestion mondiale des déchets, de mesurer l'ampleur du niveau de dégradation de la planète.

Dans la sphère idéelle, Écologie insiste sur l'importance de l'émergence d'un nouveau paradigme: le virage vert. Elle propose également d'imaginer une société où les priorités serajent renversées. C'est l'appel à l'utopie comme moteur du virage écologique.

Ces catégories d'enjeux se différencient des catégories éco-science, écodéveloppement et éco-politique déjà identifiées en tant que modes d'objectivation du monde, qui rendent difficilement compte de l'intention qui anime les médias. En fait, les catégories d'objectivation se recoupent avec diverses catégories d'enjeux. Ainsi, l'enjeu société de conservation qui se présente dans la sphère matérielle comme la recherche d'une conciliation entre progrès et écologie et dans la sphère idéelle comme la promotion d'un développement social et scientifique, concilie les trois modes d'objectivation du monde (éco-science, éco-politique et éco-développement). De même, le holisme tant matériel (donner priorité à la survie planétaire) qu'idéel (développer un nouveau paradigme) fait appel aux trois modes d'objectivation. Ce qu'il convient de noter ici, c'est que les catégories d'objectivation 
renvoient aux moyens (le quoi et le comment), que privilégient les médias, alors que les catégories d'enjeux renvoient aux orientations (le pourquoi).

Une confrontation moyens-orientations nous permet de caractériser plus précisément chacun des médias selon la sphère d'activités qu'il occupe. Ainsi, Québec Science, nettement orienté vers la conciliation du progrès économique avec l'écologie (sphère matérielle de l'approche société de conservation), préférerait un développement scientifique et un développement économique planifiés comme solution aux problèmes écologiques. Franc Nord orienté davantage vers la promotion d'idées de développement social (sphère idéelle de l'approche société de conservation) opterait pour les interventions politiques et économiques. Quant à la revue Écologie, elle irait dans toutes les directions puisque société de conservation et holisme y cohabitent d'un article à l'autre; en outre, les moyens privilégiés, qui relèvent de l'éco-politique et de l'éco-développement, n'excluent pas un point de vue critique de l'éco-science. Bref, il y en a pour tous les goûts! L'émission Aujourd' hui la science, pour sa part, s'oriente vers la promotion d'idées scientifiques (sphère idéelle de la société de conservation) et privilégie des moyens spécifiques, rationnels tels que la science est en mesure d'en développer.

Bien qu'Aujourd' hui la science ne se propose pas de parler d'écologie mais de science, il reste que ce magazine, tout comme Québec Science, représenterait bien ce qu'Auclair, Royer et Vaillancourt appellent la tendance environnementaliste: «Les environnementalistes ne critiquent pas de façon globale la société actuelle, mais seulement certains aspects de celle-ci, spécialement ses technologies. Ils ne remettent pas en cause les lieux et les rapports de pouvoir.» Les revues Franc Nord et Écologie appartiendraient plutôt à la tendance écologiste: «Les écologistes, quant à eux, veulent s'inspirer des grands principes qu'ils disent avoir découverts dans la science de l'écologie pour promouvoir une société plus juste, plus égalitaire et évidemment, en meilleure relation avec l'environnement.»Par contre, Écologie couvre un ensemble de domaines à tel point exhaustif qu'il est difficile de lui accorder une intention définie à partir de la seule analyse des enjeux. Quant à Franc Nord, ses remises en question de la société sont conciliables avec une plus grande démocratisation de la société actuelle.

Ces constats sur les enjeux soulevés ont suscité des interrogations sur le style des discours des médias. Nous cherchons à vérifier comment les orientations et les moyens s'organisent dans un certain style, présentant au lecteur, à travers le langage, un construit de la réalité sociale. Pour ce faire, nous avons conçu une grille qui rend compte du discours, c'est-à-dire tout à la fois des objectifs, des perspectives et des fonctions de ce discours (cf. tableau 1). Quatre objectifs rendent compte de l'intention du discours : informer, sensibiliser, mobiliser et légitimer. L'objectif d'informer correspond à la perspective fonctionnelle (exposer l'argumentation) et à la fonction cognitive (capacité de connaitre, de mémoriser et de nommer). Le style informatif vise essentiellement à décrire «ce qui est», il utilise la troisième personne et des verbes d'état (il est, il y a, il se passe...). L'objectif de sensibiliser relève plutôt de la perspective analytique, laquelle cerne les arguments, les hiérarchise et les positionne dans l'univers du discours. Cet objectif relève de la fonction affective (conscience, appartenance, identité, sentiments). Il se traduit dans un style orienté vers «ce qui est possible» et «ce qui s'oppose». Le «je» et le «nous» y sont utilisés de même que les constructions de cause à effet ( $\mathrm{si} \rightarrow$ donc). Quant à l'objectif de mobiliser, il relève de la perspective normative (remises en question de règles, de normes). Sa fonction est comportementale (elle concerne les devoirs, les obligations, les justifications) et se traduit par le style de «ce qui convient» (falloir, devoir). Finalement, l'objectif de légitimer, plus rarement rencontré, appartient à la perspective philosophique (principes universels), il s'articule dans une fonction éthique et morale (l'absolu et le sacré) et se concrétise au niveau du style par le «pourquoi» (visions paradigmatiques, globales et totalisantes). 
Tableau 1

Grille descriptive du discours

\begin{tabular}{|c|c|c|c|}
\hline $\begin{array}{l}\text { Objectifs du } \\
\text { discours }\end{array}$ & Perspective & Fonction & Style \\
\hline Informer & $\begin{array}{l}\text { Fonctionnelle } \\
\text { (Information sur les faits et } \\
\text { présentations des arguments) }\end{array}$ & $\begin{array}{l}\text { Cognitive } \\
\text { (Connaître, mémoriser, } \\
\text { différencier, nommer) }\end{array}$ & $\begin{array}{l}\text { Ce qui est } \\
\text { (Il est, il y a, il fait) }\end{array}$ \\
\hline Sensibiliser & $\begin{array}{l}\text { Analytique } \\
\text { (Dramatisation et hiérarchisa- } \\
\text { tion des arguments) }\end{array}$ & $\begin{array}{l}\text { Affective } \\
\text { (Sentiments, appartenance, } \\
\text { conscience...) }\end{array}$ & $\begin{array}{l}\text { Ce qui est possible } \\
\text { Ce qui s'oppose } \\
(« J e », ~ « n o u s », ~ « \text { vous», } \\
\text { «faire», «si } \rightarrow \text { donc») }\end{array}$ \\
\hline Mobiliser & $\begin{array}{l}\text { Normative } \\
\text { (Interprétation, invitation à } \\
\text { l'action et remises en question } \\
\text { de règles, de normes) }\end{array}$ & $\begin{array}{l}\text { Comportementale } \\
\text { (Obligations, justifications) }\end{array}$ & $\begin{array}{l}\text { Ce qui convient } \\
(« \text { Falloir } », \ll \text { devoir } »)\end{array}$ \\
\hline Légitimer & $\begin{array}{l}\text { Philosophique } \\
\text { (Principes universels) }\end{array}$ & $\begin{array}{l}\text { Éthique et morale } \\
\text { (Absolu, sacré) }\end{array}$ & $\begin{array}{l}\text { Le pourquoi } \\
\text { (Visions paradigmatiques, } \\
\text { globaliser, totaliser) }\end{array}$ \\
\hline
\end{tabular}

\section{OBJECTIFS DONNÉS AU DISCOURS ET STYLISATION}

L'analyse descriptive du discours des médias peut être schématisée suivant cette grille. Rappelons les quatre objectifs identifiés: informer, sensibiliser, mobiliser et légitimer. Chacue objectif se traduit dans une perspective, une fonction et un style.

Informer: l'objectif d'informer relève d'une perspective fonctionnelle (exposé des arguments), s'exprime à travers la fonction cognitive (connaître, mémoriser, différencier) et se traduit dans un style descriptif: "ce qui est». Tous les textes analysés sont animés par cet objectif mais de façons différentes.

Lorsqu'il vise à informer, Québec Science adopte une argumentation serrée. Les textes sont fortement documentés, $s$ 'appuyant sur des références d'ordre scientifique et technique et le discours des experts. Les textes sont écrits à la troisième personne et essentiellement descriptifs. Ils rendent compte de processus complexes tels l'action des polluants dans la production d'énergie, les choix mondiaux en matière de conservation d'énergie et de procédés de dépollution: «Ces organismes utilisés comme bio-indicateurs permettent de mesurer directement l'impact de la pollution sur le système écologique fluvial.» (Mc Lean Alan)

Dans Franc Nord, l'information occupe aussi un rôle central. On y retrouve des séries descriptives, énumérations, informations quantifiées sur la nature des problèmes: «Les espèces fauniques rares ou menacées au Québec fréquentent les milieux humides dans une proportion de 70 pour cent pour les poissons, de 44 pour cent pour les oiseaux et de 50 pour cent pour les amphibiens et les reptiles.» (Drapeau) Les conséquences de ces activités sont traitées rigoureusement: «Pourtant, depuis 1950, plus de 6 pour cent des habitats riverains, soit plus de 3600 hectares, ont été détruits le long du Saint-Laurent.» (Farrar)

Écologie informe, oui! mais ce n'est pas sa raison d'être: L'information n'est rigoureuse que lorsqu'on se propose de rapporter des événements précis, de présenter des personnes et des institutions. On renseigne également sur diverses industries impliquées 
dans le développement économique, leur rentabilité, leurs liens avec les divers gouvernements. On explique le concept de développement durable: ses enjeux, son origine, ses objectifs. «Faire du développement durable, c'est planifier l'économie en tenant compte des conséquences à long terme, gérer en fonction du renouvellement des ressources naturelles, de la consommation d'énergie, de l'étalement urbain.» Les concepts occupent une place modeste dans les articles et s'ajoutent à des séries d'informations générales et floues: «Cinq milliards de tonnes de ce gaz sont crachés dans l'atmosphère.» (Lemieux) Aucune allusion ici au quand, où et comment. L'information prend ainsi une allure plus impressionniste, et ce qu'on perd en rigueur, on le gagne en images éloquentes.

L'aspect informatif apparaît le plus substantiel dans le magazine Aujourd'hui la science. Les émissions sont construites sur l'information scientifique et les experts invités rendent compte de l'état de la situation dans leur domaine respectif. Les informations portent, entre autres, sur des événements, congrès, gestes posés par des équipes scientifiques:

Depuis le 7 janvier dernier, les scientifiques canadiens sont à la base militaire dans le Grand Nord canadien pour essayer de mesurer l'épaisseur de la couche d'ozone. C'est la cinquième année consécutive que les Canadiens retournent dans le Nord et ils y resteront jusqu'à la fin de février. (28 janvier 1990.)

L'aspect le plus développé des informations concerne cependant les phénomènes physiques, chimiques et biologiques:

[...] l'azote organique est d'abord hydrolysé, et peut donner de l'azote ammoniacal. Et cet azote ammoniacal, quand il y a aération, est oxydé en nitrate. Quand on passe en mode anaérobie, donc où il y a absence d'aération, les nitrates sont réduits sous forme d'azote moléculaire par une réaction qu'on appelle une réaction de dénitrification. (25 février 1990.)

Bref, si on définit l'aspect informatif selon des règles de rigueur, de pertinence et de précision, c'est à l'émission Aujourd' hui la science que revient la cote supérieure dans l'objectif d'informer. Québec Science et Franc Nord suivent, dans un style plus proche des problématiques politiques, économiques et sociales. Finalement, Écologie fait davantage appel à des généralités et des lieux communs pour traiter des questions sociales.

Sensibiliser: l'objectif de sensibiliser relève, pour sa part, d'une perspective analytique (hiérarchie des arguments). Il s'exprime à travers la fonction affective (appartenance, conscience de soi et des autres sentiments) et se traduit dans un style du «possible» et de l'«opposé». Tous les textes y font allusion.

Dans Québec Science, on rappelle au lecteur qu'il est impliqué, qu'il appartient à une communauté: "Tout n'a pas été fait pour améliorer notre efficacité énergétique. Mais au bout de la ligne, il nous faudra choisir...» (Vézina) Mais il s'agit d'un «nous» général, non précisé. De plus, le lecteur est amené à se situer dans la hiérarchie des arguments, à saisir ce qui est possible: «Ces procédés offrent peut-être la troisième voie tant désirée, bien que les projections du ministère $[. ..] \gg$ (Vézina) Tous les textes visent à faire prendre conscience des problèmes: «À l'heure du lunch, les conversations des collègues de travail, toutes axées sur la consommation de biens, vous laisseront peut-être croire que les économies d'énergie sont choses du passé.» (Parent) Ainsi, le lecteur est affectivement associé au problème posé et doit participer à l'effort d'organiser les arguments, de se faire une opinion sur la question, de développer sa conscience des problèmes.

Dans Franc Nord, on se donne aussi pour objectif de sensibiliser. L'insistance sur le «nous» (l'appartenance) est manifeste, et ce «nous» est souvent identifié à une collectivité, les Québécois, ou à un groupe (groupes environnementaux, groupe de travail ou de recherche). «Au fil des années, plusieurs grands projets dont les Québécois s'enorgueillissent encore $[\ldots] \gg$ (Drapeau)

La revue identifie également «ce qui s'oppose», par exemple : protecteur et pollueur: «Chaque Québécois n'est-il pas le propriétaire, le protecteur et l'utilisateur de ces territoires? 
Il en est aussi le pollueur, le développeur et l'envahisseur.» (Farrar); mais aussi ce qui est possible: «Néanmoins, grâce à la collaboration de la communauté, le processus de détérioration a pu être renversé et complètement freiné» (Lauzon).

De façon générale, Franc Nord axe l'objectif de sensibilisation davantage sur l'appartenance que sur la conscience. Les références à la collectivité, à la coopération, à la concertation sont des plus manifestes.

C'est dans Écologie que l'objectif de sensibilisation est le plus développé. Plusieurs informations citées dans Écologie doivent être classées dans la catégorie sensibilisation, et ce parce qu'elles sont fondées essentiellement sur des présupposés, des prévisions, des estimés. «En réalité, si le thermomètre planétaire devait grimper ne serait-ce que d'un ou deux degrés, il y aurait de quoi bouleverser la vie terrestre.» (Lemieux) De cette façon, les informations revêtent un caractère dramatique plutôt que de simplement traduire un événement. Elles sont ainsi davantage tournées vers la conscience des risques.

Afin de hiérarchiser les arguments utilisés, Écologie recourt abondamment à des exposés sur ce «qui est possible» et sur «ce qui s'oppose». Dans ce qu'il est possible de faire, on présente des idées réalisables: «On cherche donc encore des solutions efficaces, le principe du pollueur payeur en est peut-être une.» (Denis) Dans ce qui s'oppose, on insiste sur les écarts entre des volontés de faire et les gestes concrets qui sont posés, les écarts entre les gestes posés et l'ampleur des conséquences. «Les gens disent qu'ils veulent protéger l'environnement, mais est-ce qu'ils sont prêts à faire des sacrifices à ce sujet?» (Constantin) «Malgré que cette échéance puisse nous paraître lointaine et hypothétique, à force de laisser faire, elle continuera à se matérialiser dans le champ des possibles.» (Boukatem)

Dans ce périple de la sensibilisation, Écologie utilise abondamment l'expression des sentiments. La crainte, l'inquiétude, la catastrophe sont à nos portes: «Parce qu'on peut arriver, si on ne fait pas attention, à certaines irréversibilités dangereuses pour l'écosystème monclial.» (Constantin) «Sans politique environnementale, l'avenir des pays du Tiers Monde est sans issue.» (Lemieux) Quelquefois, aussi, l'espoir: «L'optimisme est peut-être un peu fort, mais bon, l'espoir est là!» (Légaré) L'expression de ces sentiments se fait toujours autour d'un hypothétique événement. La structure de phrase «si $\rightarrow$ alors» y domine. Mais parce que les relations de cause à effet portent sur des possibilités et non des événements, la croyance devient plus importante que la démonstration. Et qui dit croyance dit méfiance. «Quoi qu'il en soit, l'un des plus grands mythes de la science aura été de prétendre qu'il est possible d'éliminer les risques.» (Bélanger) En fait, ni la science ni les institutions ne trouvent grâce. Mais à qui faut-il faire confiance? Au «nous»? Ce terme est faiblement utilisé par Écologie. Il n'est jamais personnifié et demeure une généralité: «Nous pouvons agir directement» (Lacombe); «notre problème à nous aura été d'y croire» (Bélanger) «Puisque le Sud écope, il pourrait bien être en droit de nous demander des comptes» (Lemieux).

Ainsi, même si Écologie insiste beaucoup sur l'objectif de sensibiliser et que son approche analytique est développée, elle n'arrive pas à situer la fonction affective dans l'appartenarce. Tout comme Québec Science, Écologie vise davantage à la prise de conscience, mais plus que Québec Science, elle en reste à l'expression des sentiments, de la révolte, éprouvant de la difficulté à asseoir ses positions sur des mobilisations.

Dans Aujourd' hui la science, l'objectif de sensibiliser s'actualise principalement sous la forme d'une hiérarchisation des arguments où sont utilisés abondamment les «nous» et les «on», lesquels renvoient généralement à la communauté scientifique ou aux activités d'un groupe de chercheur. À l'occasion, le «nous» peut désigner l'ensemble de la société.

L'aspect affirmatif de l'argumentation transparaît à travers le traitement de «ce qui est possible» ou de «ce qui s'oppose». L'objectif est souvent d'affirmer qu'une approche est supérieure à l'autre, qu'elle vaut par la rigueur de sa démonstration: «L'avantage de cette méthode, de ce phénomène, c'est qu'on peut mesurer en effet un stress chimique très vite et la méthode peut être très fiable; et c'est pas cher aussi et on peut contrôler notre environnement de façon très rationnelle et en continu.» (4 février 1990.) 
Dans le processus d'organisation de l'argumentation, l'émission insiste également sur l'opposition entre différentes approches. Elle cherche à en clarifier les contenus et à en définir les conséquences. C'est l'aspect discussion de l'argumentation: «Un des problèmes quand on parle de biodégradabilité du plastique, Yannick, c'est qu'on ne s'entend pas sur une durée pour ce processus, ni sur les conditions dans lesquelles il doit se dérouler.» (28 janvier 1990.)

L'expression des sentiments n'est pas absente du discours, quoique faiblement utilisée. On fait référence à l'urgence d'agir: «Et pourtant dans beaucoup de cas le temps presse» (11 mars 1990) aux craintes: «Évidemment, les autorités médicales se sont inquiétées» (18 mars 1990); au danger: «Oui, le danger existe en effet» (18 mars 1990). Mais, même sur les sentiments, la discussion existe: «C'est peut-être extrêmement inquiétant, comme ça peut très bien être un effet, si vous voulez, de la construction un petit peu au hasard.» (18 mars 1990.)

La sensibilisation se fait aussi par une insistance sur «ce qui s'oppose». Tantôt on décrit les faits et l'action gouvernementale: «Devant un tel état de faits, pourquoi nos représentants élus se lancent-ils tête baissée dans un tel projet?» (8 janvier 1990); tantôt on souligne l'incongruité des actions entre cueillette et recyclage: «Pourquoi empiler des contenants de verre, de plastique et des journaux quand on ne sait même pas où les envoyer par la suite?» (9 mars 1990); tantôt on met l'accent sur la richesse et le gaspillage : «C'est qu'il est riche le Québec qui se paie un stade d'un milliard. Pas nécessaire quand même de dilapider l'argent des contribuables» (21 mars 1990).

De tous les médias, il revient à la revue Écologie d'assumer avec le plus d'intensité la fonction de sensibilisation. La confrontation des idées et des faits, les possibles toujours à réinventer, les sentiments d'inquiétude répétés, de même que le «nous» général en font une revue dans laquelle l'appartenance est mal définie et où la conscience des problèmes est exacerbée, mais inapte à s'inscrire dans un projet social. À l'inverse, Aujourd' hui la science définit son appartenance à la communauté scientifique, y situe les possibles et les oppositions, traçant ainsi un lieu d'intervention avec ses objectifs et ses limites. Il n'y a pas ici d'ambiguïté d'appartenance, bien que celle-ci soit restreinte. La revue Franc Nord parvient à inscrire son identité dans la collectivité québécoise, son intention vers une plus grande intégration qui passe par la coopération et la concertation. Conséquemment, sa capacité analytique apparaît intégrée et sa fonction affective assumée. Dans Québec Science, le «nous» reste imprécis et général. L'objectif est davantage tourné vers la conscience des problèmes que vers l'appartenance, ce qui donne à son projet social un aspect plus «intellectuel».

Mobiliser: l'objectif de mobiliser relève d'une perspective normative (remise en question des règles et des normes). Il s'exprime à travers la fonction comportementale (obligations, justifications) et se traduit dans le style de «ce qui convient». Cet objectif n'est pas repris dans tous les textes et articles. Dans Québec Science, deux textes le remplissent. On insiste sur ce qu'il faut faire : «On doit tout simplement s'habituer à appliquer une multitude de petites mesures [...]» (Parent) Ces propositions s'acccompagnent de remises en questions de comportements et de procédés. «L'approche scientifique consistant à mesurer la pollution en parties par millions est totalement dépassée.» (McLean) De façon générale, les remises en question portent sur les façons de penser le monde. Les modifications de comportement découleront du changement des modes de pensée.

Dans Franc Nord, l'objectif de mobilisation, quoique présent dans deux textes sur quatre, est traité rapidement. On utilise le «devrait» pour spécifier le rôle et la fonction des groupes de protection de l'environnement: «Tout comme il s'impose de poursuivre la concertation entre les groupes environnementaux et le gouvernement $[\ldots]$ (Drapeau)

À l'inverse, Écologie, fait fréquemment appel à la mobilisation. L'objectif de mobiliser est présent dans onze articles sur douze. Cependant, il est peu étayé dans chacun des articles. Dans la plupart des cas, il est centré autour de la nécessité d'accroître la conscience, la compréhension des problèmes: "Ce qu'il faut faire, c'est essayer de comprendre la nature et l'importance des défis écologiques qui sont devant nous» (Constantin); «Au contraire, 
le développement durable exige une vision d'ensemble, des mesures globales et du courage politique.» (Denis) À d'autres occasions, la mobilisation se fait autour de projets globaux, de visions larges et généralisées: «Il faudra donc que le Sud évite de reproduire les erreurs commises par le Nord.» (Légaré)

Au chapitre de la mobilisation, le magazine Aujourd' hui la science propose certaines remises en question tout comme il force certains choix. Ainsi, certaines formulations affirment sans ambages ce qu'il convient de faire: «Il faut agir vite, au moins pour répondre à l'anxiété [...] (18 mars 1990), alors que d'autres proposent la prudence dans l'action. «Mails il faudra bien faire attention de choisir le créneau approprié» (28 janvier 1990), ou insistent sur l'importance de comprendre: «Il faut bien comprendre que chaque année, il y a une production entre 8 et 10000 nouveaux produits chimiques» (4 février 1990). Le magazine accorde, en fait, beaucoup moins d'importance à l'objectif de mobiliser qu'à l'information et à la sensibilisation.

L'objectif de mobiliser est fortement lié à celui de sensibiliser. Les médias qui centrent leur activité sur la sensibilisation font de même pour la mobilisation, et surtout ils le font dans le même esprit. Ainsi, l'activité de mobilisation dans Écologie est, elle aussi, centrée sur une conscience et une compréhension accrues des problèmes environnementaux. Sa difficulté à définir son appartenance l'oblige toutefois à centrer la mobilisation sur des projets ou visions larges des problèmes analysés. Aujourd'hui la science, pour sa part demeure localiste. Son appartenance à la communauté scientifique se traduit par l'affirmation des règles et normes propres au travail scientifique. La recherche de la solution appropriée à chaque situation singulière est associée à un exercice préalable de compréhension. Franc Nord, fidèle à son appartenance collective, oriente la mobilisation vers les activités proposées par les groupes environnementaux, alors que Québec Science accentue son projet de création d'une conscience collective apte à produire des modifications de comportement.

Légitimer: l'objectif de légitimer relève d'une perspective philosophique (principes universels). Il s'exprime à travers la fonction éthique ou morale (l'absolu et le sacré) et se traduit dans le style du «pourquoi». Cet objectif est nettement moins présent dans les articles et les émissions radiophoniques. Seule la revue Écologie lui accorde une importance significative. Cinq articles sur douze y réfèrent. Sur ce thème, on traite de globalisation de l'action, de nouveau paradigme: «Nous disons qu'il faut imaginer une société [...]» (Constantin), «que le virage vert ne sera pas effectif, chaque fois réaffirmé et ancré dans chacune de nos décisions» (Boukatem). Un texte va plus loin dans l'univers éthique et moral: «Hors de l'implication point de salut.» (Turcotte) Ainsi, la vision paradigmatique sert davantage à légitimer l'orientation de la revue vers une plus grande conscientisation qu'elle n'est un véritable exercice philosophique.

Dans Québec Science, un seul texte réfère à l'objectif de légitimer. Il pose la nécessité de dépolluer dans une perspective d'absolu de la vie: «[...] mais une chose est certaine, la vie peut renaitre de la mort. » (McLean) De même, dans Aujourd' hui la science, cet objectif est relativement absent. Un exposé y renvoie toutefois, insistant sur l'éthique: «Parce que l'objectif de la recherche scientifique c'est l'objectif de la vérité. Trouver la vérité...» (25 mars 1990) II faut noter que cet article est fortement polémique et rejette des hypothèses de plus en plus admises: la destruction de la couche d'ozone et l'effet de serre. Dans le déséquilibre qu'une telle opposition suppose, la recherche d'une légitimité est sans doute plus importante que dans le cadre d'un simple débat sur l'âge des poissons utilisés pour établir les bio-essais. À ce titre, la fonction de légitimer ne semble apparaitre que lorsqu'il convient de justifier le débat.

En résumé, Québec-Science aborde les questions écologiques à travers une fonction principalement cognitive, sa fonction affective étant orientée vers la conscience des problèmes, laquelle assure l'intégration des connaissances dans le monde vécu du lecteur. Si la revue Québec Science se donne à l'occasion des objectifs de mobilisation et de légitimité, elle ne vise somme toute qu'à montrer la nécessité de changer les idées. Dans Franc Nord, la fonction cognitive apparaît manifeste. Elle trouve cependant une intégration 
affective dans le sentiment d'appartenance plutôt que dans le développement de la conscience. De cette façon, l'objectif d'informer devient partie prenante du désir de former une pensée collective, un univers communicationnel intégrateur. À l'opposé de Franc Nord, qui met l'accent sur l'activité cognitive pour réaliser une intégration affective, la revue Écologie cherche à ancrer son travail dans la fonction affective pour déboucher sur une intégration cognitive qui dirigerait les comportements et les modes de vie. Dans tous les cas, le point de vue n'est pas «neutre» mais partisan et vise à intervenir sur les normes et les règles qui régissent le fonctionnement social. Aujourd' hui la science se caractérise par son discours orienté vers les contenus scientifiques des sciences de la nature et par son insistance sur l'argumentation: rigueur de l'exposition et contre-arguments. Cependant, l'émission se préoccupe peu de trancher les questions d'ordre social. Plutôt, elle oriente le débat sur les principes évolutifs de la science elle-même, de la recherche scientifique et du développement des technologies. Le savoir «se faisant», voilà à quoi nous convie l'émission lorsqu'elle traite des questions écologiques. La fonction affective se matérialise donc dans la conscience des problèmes, le contrôle par l'intelligence, renforçant ainsi l'importance que l'émission accorde à la fonction cognitive.

\section{PROBLÈMES, ENJEUX ET DISCOURS}

L'analyse des problèmes a permis d'identifier les différentes façons d'objectiver les questions écologiques dans les médias. Les variations observées ne peuvent que dériver de l'intention qui fonde chacun des médias. L'analyse des enjeux et du discours nous permet de confirmer une telle hypothèse. Ainsi, si Aujourd' hui la science insiste tant sur les questions de pollution, c'est que cette thématique recouvre des problèmes facilement décomposables en éléments restreints, concrets et qui donnent prise à l'approche scientifique et aux technologies. Les problèmes de gaspillage des ressources, peu ou pas abordés par Aujourd' hui la science mais très présents dans le discours écologique, sont pour leur part toujours plus généraux et font intervenir d'abord et avant tout une problématique sociale de la pertinence. À partir de quel seuil peut-on parler de gaspillage? Où se situe le point limite entre exploitation et sur-exploitation? Autant de questions qu'une approche scientifique qui se définit par la précision, la rigueur et la compétence dans l'identification du problème, lequel doit faire l'objet de solutions technologiques concrètes, peut difficilement aborder. L'approche «gaspillage des ressources» relève davantage des sciences humaines qui, intervenant dans la formulation des règles et normes, peuvent rendre compte des effets, des conséquences et des possibilités entourant une situation donnée. En fait, l'approche scientifique de l'émission Aujourd' hui la science est une approche nettement orientée vers les sciences de la nature et leur pendant pratique: les technologies.

En outre, si Franc Nord reprend à son compte la thématique du gaspillage des ressources, c'est que son propos touche la pertinence de l'action sociale. Franc Nord cherche à développer l'intégration sociale autour d'un projet de société. Les problématiques de gaspillage permettent le questionnement des valeurs, objectifs et orientations sociales. En ce sens, on pourrait s'attendre à ce que Franc Nord développe davantage les aspects de la thématique «planète en danger», plus propice à ce type de questionnement. Il est probable que l'explication de ce silence se trouve dans la mission scientifique de Franc Nord, laquelle l'oriente vers la sphère idéelle mais à l'intérieur d'une société de conservation, dans laquelle les solutions sont possibles.

Quant à la revue Québec Science, elle est à la fois déterminée par sa vocation scientifique et par son ancrage dans une dynamique de conscientisation sociale. Son propos n'étant pas de contribuer à la construction de l'identité sociale de ceux qui se mobilisent pour la cause écologique, elle est à l'aise dans les débats sur les questions de pollution et même de gaspillage dans la mesure où ils renvoient aux conséquences de la pollution.

Ecologie, pour sa part, semble s'être donné pour mandat de mieux faire prendre conscience des problèmes. Cependant, la revue s'éparpille entre tous les enjeux possibles et 
son discours «sensible»s'arrime difficilement aux institutions, aux organisations de la collectivité à laquelle il s'adresse.

En fait, il apparaît difficile pour un média de pousser très loin la problématique écologique s'il vise seulement à conscientiser. À cet égard, la revue Franc Nord est sans doute celle qui est la plus proche d'un véritable discours écologique, en ce que son propos renvoie à l'impératif de construction d'une nouvelle culture qui créerait un sentiment d'appartenance. Sa difficulté semble être de concilier science et action sociale.

Quoi qu'il en soit, ce que cette analyse des représentations met en évidence, c'est que les médias présentent une objectivation de la problématique de l'écologie à partir d'une intention préalable. Cette intention définit le média, intervient dans le choix des thématiques abordées et en oriente le traitement. C'est en ce sens que le média joue un rôle d'analyste, qu'il est producteur de sens et de savoir sur le monde. L'activité des médias constitue un bon exemple de la construction du social à travers la communication.

\section{CONCLUSION}

Au terme de cette analyse, il apparaît donc que les éléments du discours scientifique sont intégrés dans un discours médiatique sur le social, lequel se présente à travers une articulation d'enjeux et d'objectifs spécifiques à chaque média. Les discours sur les dangers pour la planète, la pollution, le gaspillage et le développement durable cherchent à définir le «comment» des problèmes écologiques. Ils sont présentés tantôt à travers une approche réductionniste (l'approche éco-science qui dissèque la réalité en problèmes et solutions spécifiques participe de ce modèle), tantôt de façon globaliste (l'approche éco-politique utilise ce procédé pour rattacher les problèmes écologiques à des objectifs politiques plus globaux), ou encore à travers une approche intermédiaire centrée sur la recherche de principes et d'orientations, qui permettent d'utiliser des outils économiques spécifiques à des situations sociales plus vastes: l'approche éco-développement est de cet ordre.

Ces aspects nous informent sur l'aspect «objectivation» des représentations. Ils mettent en évidence ce qui est nommé, ce qui est sélectionné pour fournir la représentation des problèmes écologiques. Ces construits représentationnels organisés à travers différents modèles (éco-science, éco-politique, éco-développement) permettent le tri et l'organisation des informations. Ainsi les problèmes que soulève la pollution peuvent être présentés dans un média à travers une approche «éco-science» où des problèmes spécifiques appellent des solutions spécifiques. C'est la démarche qu'utilise Aujourd' hui la science. Les problèmes de pollution peuvent aussi être présentés à travers une approche «éco-politique» ou «éco-développement» et donner lieu à une généralisation à l'ensemble des problèmes économiques ou planétaires, lesquels ouvrent sur un éventail particulièrement large de problèmes et de solutions de moins en moins spécifiques. L'approche éco-politique des questions de pollution serait davantage le fait de la revue Écologie, alors que celle d'écodéveloppement pourrait être davantage rattachée à Québec Science. Quant à Franc Nord, la revue construit une représentation qui relève tantôt de l'éco-politique, tantôt de l'écodéveloppement, et ce sur les questions de gaspillage des ressources exclusivement.

En ce sens, les représentations existent à partir de modèles qui viennent sélectionner, organiser et structurer l'information de façon à la rendre intelligible. Ce «comment» de la représentation constitue un point de départ de la compréhension des représentations mais ne peut l'expliquer totalement. Le questionnement sur le «pourquoi», que nous avons poursuivi à travers l'analyse des mécanismes d'ancrage, a cherché à situer les représentations dans leurs réseaux de significations, à définir la valeur fonctionnelle des savoirs qui y sont inclus.

Ainsi, l'analyse des enjeux a permis de montrer que les revues situent les enjeux du débat sur l'écologie dans les structures sociales elles-mêmes. Les problèmes écologiques n'ont de sens, dans les médias, qu'en ce qu'ils constituent des problèmes sociaux : problèmes sociaux pour les détenteurs du pouvoir, pour les citoyens ou pour les institutions elles-mêmes. 
L'identification des intentions sous-jacentes au discours (informer, sensibiliser, mobiliser et légitimer) nous a fourni un outil de compréhension des mécanismes d'ancrage des représentations. Cette analyse a permis de cerner le fait que certains médias poursuivaient des objectifs davantage centrés sur la vulgarisation: connaître pour transformer. D'autres cherchent principalement à développer à partir de faits objectifs un sentiment d'appartenance et de responsabilité sociale. En fait, Québec Science et Aujourd' hui la science appartiendraient davantage au premier groupe, alors que les revues à mandat écologique tablent davantage sur la fonction affective, tantôt pour exacerber la conscience (Écologie), tantôt pour raffermir l'identité collective (Franc Nord). Puisque l'objectivation et l'ancrage relèvent d'aspects du monde distincts (le «comment» et le «pourquoi»), leur articulation peut donner lieu à une multiplicité de styles. En ce sens, chaque média se distingue de l'autre dans la division du travail médiatique par le choix des dominantes qu'il choisit de faire ressortir. Si l'analyse des représentations sociales nous autorise à qualifier l'«esprit de l'écologisme» qui règne dans certains médias au Québec, nous pouvons affirmer que cet esprit est informé par une hybridité singulière qui conjugue la rationalité scientifique avec une normativité de l'action. Cette hybridité nous permettra ultérieurement de réexaminer le statut des médias dans leur travail de vulgarisation scientifique.

\author{
Brigitte Dumas \\ et Carmen Gendron \\ Département de sociologie \\ Université de Montréal \\ C.P. 6128 , Succ. «A» \\ Montréal (Québec) \\ Canada $\mathrm{H} 3 \mathrm{C}$ 3J7
}

\title{
BIBLIOGRAPHIE
}

ABRIC, J.-C. (1984), «L'artisan et l'artisanat: analyse du contenu et de la structure d'une représentation sociale», Bulletin de psychologie, vol. 37, p. 861-875.

ABRIC, J.-C., R. MARDELLAT (1974), «Étude expérimentale des représentations dans une situation conflictuelle: rôle du contexte de la tâche, de la place et de la pratique des sujets dans la formation sociale», Bulletin de psychologie, vol. 27, $\mathrm{n}^{\text {or }} 1-4, \mathrm{p} .146-152$.

ABRIC, J.-C., G. VACHEROT (1975-1976), «Méthodologie et étude expérimentale des représentations sociales: tâche, partenaire et comportement en situation de jeu», Bulletin de psvchologie, vol. 29, p. 735-746.

ACKERMAN, Werner, Renaud DULONG (1971). «Un nouveau domaine de recherche : la diffusion des connaissances scientifiques», Revue française de sociologie. vol. 12, p. 378-405.

ACKERMAN, W., R. ZYGOURIS (1973-1974), «Représentation et assimilation de connaissances scientifiques», Bulletin du CERP, vol. 22, p. 1-130.

ALBERTINI, Jean-Marie, Claire BÉLISLE (1988), Les Fonctions de la vulgarisation scientifique et technique. Vulgariser la science, le procès de l'ignorance, (sous la dir. de Chanvallon), Jacobi et Schiele.

ALBERTINI, Jean-Marie (1984), Représentations et initiation scientifique et technique. Les Savoirs dans les pratiques quotidiennes: recherches sur les représentations (sous la dir. de C. Bélisle et B. Schiele), Paris, CNRS,

ARNAULT de LA MÉNARDIÈRE, M. A., DE MONTMOLLIN, G. (1984), «La représentation comme structure cognitive en psychologie sociale», Psychologie française, vol. 30 , p. 239-244.

AUCLAIR, S., J. ROYER, J.-G. VAILLANCOURT (1990), Trois Revues québécoises entre l'environnementaliste et l'écologisme, communication au Congrès de l'ACFAS, mai, à paraître.

BÉLISLE, Claire, Bernard SCHIELE (1984), «Conclusion: la résurgence du concept», Les Savoirs dans les pratiques quotidiennes, sous la dir. de C. Bélisle et B. Schiele) CNRS Paris.

CODOL, J.-P., C. FLAMENT (1971), «Représentations de structures sociales simples dans lesquelles le sujet est impliqué», Cahiers de psychologie, vol. 14, p. 203-128.

CODOL, J.-P. (1970), «La représentation du groupe, son impact sur le comportement des membres d'un groupe et sur leurs représentations de la tâche, d'autrui, et de soi», Bulletin de psvchologie, vol. 24, p. 11-122.

DENIS, M., D. DUBOLS (1976), «La représentation cognitive: quelques modèles récents», L'Année psychologique, vol. 76, p. 541-562.

DI GIACOMO, J.-P. (1981), «Aspects méthodologiques de l'analyse des représentations sociales», Cahiers de psychologie cognitive, vol. 1, p. 397-422. 
DOISE, W. (1985), «Psychologie sociale et constructivisme cognitif», Archives de psychologie, vol. 53, $\mathrm{n}^{\circ} 204$, p. $127-140$

DOISE, W. (1985), «Les représentations sociales: définition d'un concept», Connexions, vol. 45, p. 245-253.

FARR, Robert (1987), "Social Representations: A French Tradition of Research», Journal for the Theory of Social Behaviour, vol. $17, n^{\circ} 4$, p. 343-369.

FARR, Robert, MOSCOVICI, Serge (dir.) (1984), Social Representation. Cambridge, Cambridge Univerversity Press.

FLAHAULT, François (1982), «Sur le rôle des représentations supposées partagées dans la communication», Connections, vol. 43 , p. 30-37.

FLAMENT, C. (1981), «Sur le pluralisme méthodologique dans l'étude des représentations sociales», Cahiers de psychologie cognitive, vol. 1, p. 423-429.

GLASIER, Robert (1984), «La science cognitive et l'éducation», Revue internationale des sciences sociales, Unesco, p. 23-25.

GRIZE, Jean Blaise, Pierre BERGES, A. SILEM (1987), Salariès face aux nouvelles technologies: vers une approche sociologique des représentations sociales, Paris. CNRS, p. 219

HEWSTONE, M. (1984), «On Common Sense and Social Representation», British Journal of Social Psychology, vol. 24 , p. $95-97$.

JODELET, Denise (1989), «Représentations sociales; un domaine en expansion», Les Représentations sociales, P.U.F., Paris.

LE BOUEDEC, G. (1984), «Contribution à la méthodologie d'étude des représentations sociales», Cahiers de psychologie cognitive, vol. 4, p. 245-272.

LIPIANSKY, E. Marc (1987), «Langage, représentations et identité culturelle», Amis de Sèvres, vol. 125, p. $60-88$.

MOSCOVICI, Serge (1988), «Notes Towards a Description of Social Representations, European Journal of Social Psychology, vol. 18, p. 211-250.

MOSCOVICI, Serge (1987), Answers and Questions. Special Issue: Social Reprepsentations. Journal of the Theory of Social Behaviour, vol. 17, $\mathrm{n}^{\circ} 4$, p. 513-529.

MOSCOVICI, Serge (1984), «The Myth of the Lonely Paradigm: A Rejoinder», Social Research, vol. 51, p. 939-968.

MOSCOVICI, Serge (1976), «Psychologie des représentations sociales», Cahiers Vilfredo Pareto, vol. 14, p. $409-416$.

MOSCOVICI, Serge (1961), La Psychanalyse, son image et son public, PARIS, P.U.F.

OPPENHEIMER, R. (1971), "Réflexions sur la science et la culture», Recherches et débats du CCIF, n 39. Cité dans Ackerman (1971).

ROISIER, M. (1987), "Common Sense, Science and Public Opinion», Journal for the Theory of Social Behavior. vol. $17, n^{\circ} 4$, p. $411-431$.

\section{RÉSUMÉ}

Cet article questionne la participation des médias à la construction des représentations sociales de l'écologie dans la société québécoise. Le contenu des textes de trois revues québécoises et d'une émission radiophonique y est analysé à travers une problématique des représentations sociales, laquelle fait une large place aux concepts d'objectivation et d'ancrage. Par l'objectivation, les informations sont sélectionnées, triées et organisées en vue de présenter une vision du monde. Les mécanismes d'ancrage scrutent les objectifs, l'intention et la perspective qui rendent les éléments compréhensibles les uns par rapport aux autres. L'article identifie ainsi les problèmes et solutions proposées par les médias et lie leurs choix aux perspectives qui fondent le discours pour dégager le rôle de producteurs de sens et de savoir qu'assument les médias en matière d'écologie.

\section{SUMMARY}

This paper questions the participation of the media in the construction of social representations of ecology in Quebec society. The content of articles in three Quebec journals and of a radio program is examined from the analylical perspective of social representations which leaves a large place to the concepts of objectivization and anchoring. Through objectivization, information is selected, sorted and organized with a view to presenting a world vision. The mechanisms of anchoring scrutinize the objectives, the intention and the perspective that makes the elements comprehensible in relation to one another. In this way, this paper identifies the problems and the solutions proposed by the media and links their choice to perspectives which underlie the discourse, in order to determine the role of producer of meaning and of knowledge which the media assume in the issue of ecology.

\section{RESUMEN}

Éste artículo pone en cuestión la participación de los medios de comunicación de masas en la construcción de las representaciones sociales de la ecología dentro de la sociedad quebequence. El contenido de los textos de tres revistas quebequences y de una emisión radiofónica son analizados a travéz de una problemática de las 
representaciones sociales la cual da un amplio lugar a los conceptos de objetivación y de ancraje. Por la objetivación las informaciones son seleccionadas, clasificadas y organizadas en vista de presentar una visión del mundo. Los mecanismos de ancraje escrutan los objetivos, la intención y la perspectiva que transforman los elementos en comprensibles los unos en relación con los otros. El artículo identifica de esta manera los problemas y las soluciones propuestas por los media y liga su elección a las perspectivas que fundamentan el discurso para desprender el rol de productor de sentidos y de saberes que asumen los media en materia de ecología. 